\title{
EFECTO DE MOMENTO DE COSECHA Y PERMANENCIA EN HUERTO SOBRE LA CALIDAD EN POSCOSECHA DE ARÁNDANO ALTO (VACCINIUM CORYMBOSUM L.), CVS. BERKELEY, BRIGITTA Y ELLIOTT DURANTE LA TEMPORADA 2005-2006
}

\author{
EFFECT OF TIME TO HARVEST AND PERMANENCE IN THE ORCHARD \\ ON POSTHARVEST QUALITY IN HIGH BLUEBERRY (VACCINIUM \\ CORYMBOSUM L.), CVS. BERKELEY, BRIGITTA ELLIOTT \\ DURING THE SEASON 2005-2006
}

\author{
Daniel Figueroa $S .{ }^{1}$; Jaime Guerrero $C .{ }^{1}$; Emma Bensch $T .{ }^{1}$
}

\section{RESUMEN}

Los objetivos de este estudio fueron: evaluar efecto del momento de cosecha y permanencia en huerto almacenaje refrigerado sobre el deterioro de arándano cultivares Berkeley, Brigitta y Elliott durante la temporada 2006, cuantificar contenido de sólidos solubles y su evolución en relación con los tratamientos, cultivares y período de almacenaje en frío y laboratorio y evaluar deshidratación en función de los tratamientos, cultivares y período de almacenaje en frío y laboratorio. El estudio se realizó durante los meses de enero y febrero de 2006 en la localidad de Freire, Región de La Araucanía. Para la realización de este estudio se utilizaron frutos de maduración temprana (Berkeley) media (Brigitta) y tardía (Elliott), cosechando la fruta por la mañana entre las 9 y 11 horas y por la tarde entre las 13 y 16 horas, para luego de 2 y 4 horas de permanencia en huerto ser depositada en cestillos de exportación y realizar las evaluaciones al cabo de 14 días en cámara de frío. Los datos obtenidos fueron sometidos a un análisis de contrastes ortogonales, y las medias de los tratamientos y subtratamientos se compararon a través de pruebas de significancia mínima (LSD) ( $\mathrm{p} \leq 0.05)$. El contenido de sólidos solubles ( ${ }^{\circ}$ Brix) no presentó variación en ninguno de los cultivares con respecto al momento de cosecha y al período de permanencia en huerto, la pérdida de peso fue mayor al realizar la cosecha por la tarde, en tanto que el período de permanencia en huerto provocó una mayor pérdida de peso en poscosecha. Asimismo, la mayor deshidratación se registró en el cultivar Berkeley, seguido de Brigitta y Elliott en forma decreciente.

Palabras clave: Vaccinium corymbosum, poscosecha, sólidos solubles, deshidratación.

\begin{abstract}
The objectives of this study were to assess the effect of harvest time and permanence in orchard on the deteriorating blueberry cultivars Berkeley, Brigitta and Elliott during the 2006 season, quantifying the content of soluble solids and its performance in relation to the treatments, cultivars and period of cold storage and laboratory and evaluating dehydration depending on the treatments, and cultivars period of cold storage and laboratory. The study was conducted during the months of January and February 2006 in the town of Freire, La Araucania. For this study there were used fruits of early maturing (Berkeley) media (Brigitta) and late (Elliott), reaping the fruit in the morning between 9 and 11 am, and in the afternoon between 1 and 4 pm, so that after 2 or 4 hours of staying in the garden they were placed in export baskets and their behavior was assessed after 14 days in the cold chamber. The data were subjected to an analysis of orthogonal contrasts, and the average obtained in the treatments and subtreatments were compared through evidence of minimal significance $(L S D)(p \leq 0.05)$. The content of soluble solids $\left({ }^{\circ}\right.$ Brix $)$ was not changed in any of the cultivars with respect to the time of harvest and the period of staying in the garden, weight loss was greater when harvest is carried out in the afternoon, while the period of staying in the garden led to a greater weight loss in post-harvest, also the largest dehydration occurred in the cultivar Berkeley, followed by Brigitta and Elliott in a decreasing order.
\end{abstract}

Key words: Keywords: Vaccinium corymbosum, postharvest, soluble solids, dehydration.

1 Universidad de La Frontera, Facultad de Ciencias Agropecuarias y Forestales. E-mail: figueroa.dan@gmail.com; jguerre@ ufro.cl; eabensch@ufro.cl

Fecha de Recepción: 25 Octubre 2007

Fecha de Aceptación: 15 Enero 2008 


\section{INTRODUCCIÓN}

La poscosecha de la fruta se define tradicionalmente tanto por aspectos estéticos como textura (firmeza, jugosidad y turgencia) y la apariencia (color, frescura y ausencia de pudrición o desórdenes fisiológicos). Si bien estos términos son parte importante del concepto de calidad, se ignoran los valores nutricionales y organolépticos, siendo estos últimos los que influyen mayormente en la selección del producto por parte del consumidor y determinan de modo importante el consumo de frutos y otros alimentos (Pelayo et al., 2001). Según Yommi y Godoy (2002), a 4 y $5{ }^{\circ} \mathrm{C}$ tienen una tasa respiratoria considerada baja a moderada, pero la misma se eleva considerablemente a temperatura ambiente. Cuanto mayor es la tasa respiratoria, más rápido se producen los cambios involucrados en la maduración y en la pérdida de la calidad.

Los arándanos son frutos muy perecederos, debido principalmente a una tasa respiratoria elevada (Kader, 1992; Moggia, 1991; Salunkhe et al., 1991). En general se considera que el arándano es climatérico, denominando de este modo a aquellos frutos que presentan un marcado aumento en la actividad respiratoria (Darnell et al., 1992), el cual se produce con posterioridad a la cosecha si son recolectados con madurez fisiológica (Beattie y Wade, 1997).

Durante el proceso de maduración de las frutas, el cambio cuantitativo más importante es la casi total conversión del almidón en azúcares, ya que a lo largo de este proceso se altera tanto el gusto como la textura de la fruta (Wills et al., 1985). Los sólidos solubles están constituidos principalmente por azúcares como glucosa y fructosa y en menor grado por ácidos orgánicos y sustancias pécticas (Auda, 1973). En arándano, glucosa y fructosa son los principales azúcares, encontrándose pequeñas cantidades de sacarosa (Moggia, 1991).

Los sólidos solubles indican el porcentaje de azúcar contenido en la fruta (Wills et al., 1985). Para cosechar frutas de arándano, éstos deben presentar niveles de sólidos solubles en rango de 12 a $14^{\circ}$ Brix. Al respecto, Galleta e Himelrick (1989) postulan que los frutos maduros pueden alcanzar valores de $15^{\circ}$ Brix. En este sentido, Kushman y Ballinger (1968) proponen como criterio de cosecha, niveles superiores a $10^{\circ}$ Brix, en tanto que Lobos (1988) indica que frutos de arándano con 11 y $12^{\circ}$ Brix reúnen las cualidades organolépticas deseadas.
La mayoría de los azúcares son procesados en las hojas y luego transportados hacia el fruto; por ello, aquellos frutos madurados fuera del arbusto sólo alcanzan un contenido final de $10 \%$ de azúcar (Olivos, 1995).

La deshidratación del arándano en poscosecha genera pérdida de peso, flacidez y ablandamiento, lo que afecta directamente el valor comercial de este fruto. Una pérdida de peso de 3 a $5 \%$ del peso inicial origina arrugamiento en la fruta (Fundación Chile, 1989), bastando un 5\% de pérdida de peso por deshidratación para obtener arándanos con poca apariencia atractiva (Fundación Chile, 1986).

Para evitar las pérdidas por deterioro de la fruta en poscosecha, es indispensable la aplicación de prefrío rápido a la fruta después de cosechada (Sudzuki, 1983). Por otra parte, Guerrero (1993) indica que el arándano se conserva en buenas condiciones durante 15 a 33 días de almacenaje, en cámaras de frío a $0{ }^{\circ} \mathrm{C}$ y con $90 \%$ de $\mathrm{HR}$, dependiendo del cultivar y estado de madurez.

\section{MATERIALES Y MÉTODOS}

El ensayo se realizó en Agrícola San Judas Tadeo, huerto de arándanos de 10 años de edad; distante a $5 \mathrm{~km}$ de la localidad de Freire y ubicada en las coordenadas $37^{\circ} 57^{\prime}$ de longitud Sur y $72^{\circ} 35^{\prime}$ de latitud Oeste, con fruta cosechada en forma manual el 21 de enero de 2006 para el cultivar Berkeley; el 27 de enero de 2006 para el cv. Brigitta y el 2 de febrero para el cv. Elliott.

El lugar de estudio se encuentra en la serie de suelo Freire, la cual se caracteriza por presentarse en forma de depósitos de cenizas volcánicas descansando sobre arenas y/o gravas de las antiguas terrazas aluviales del río Toltén. Son suelos moderadamente profundos y color pardo oscuro, textura franco limosa y gravas con fierrillo en la zona de contacto con el suelo. La permeabilidad es moderada, de drenaje imperfecto y topografía casi plana con 1 a $3 \%$ de pendiente (CIREN, 2002).

El clima en esta zona es templado, el régimen térmico se caracteriza por temperaturas que varían, en promedio, entre una máxima de enero de $24,1^{\circ} \mathrm{C}$ y una mínima de julio de $4,1^{\circ} \mathrm{C}$. El período libre de heladas es de 215 días, con un promedio de 14 heladas por año. Registra anualmente 1.142 díasgrado y 1.574 horas de frío. El régimen hídrico presenta un precipitación media anual de $1.342 \mathrm{~mm}$, 
un déficit hídrico de $439 \mathrm{~mm}$ y un período seco de 3 meses (Santibáñez y Uribe, 1993).

\section{TRATAMIENTOS}

El tratamiento considerado fue el momento de cosecha de la fruta, por la mañana (9-11 hrs.) y por la tarde (13-16 hrs.), en tanto que los subtratamientos correspondieron al intervalo de tiempo entre la cosecha y puesta en frío de la fruta, siendo de 2 y 4 horas para cada tratamiento.

\section{COSECHA}

Como criterio de cosecha se utilizó el color azul de cubrimiento de las bayas, cosechando $2,5 \mathrm{~kg}$ de fruta para cada tratamiento. A la cosecha se tomaron 10 frutos por cada subtratamiento, a los cuales se le determinó su contenido de sólidos solubles. En cada día y hora de cosecha se registró la temperatura y humedad ambiental.

Una vez cosechada la fruta, se depositó en cajas tipo clamshell con $125 \mathrm{~g}$. de capacidad (110 frutos aproximadamente); cada cestillo fue pesado y registrado previo a su almacenamiento en cámara de frío. Los clamshell con fruta fueron dispuestos en bandejas de exportación de 12 unidades para su ingreso a almacenaje refrigerado $\left(0{ }^{\circ} \mathrm{C}\right.$ y $\left.90 \% \mathrm{HR}\right)$ durante 14 días, al cabo de los cuales la fruta fue sometida a evaluación.

\section{EVALUACIÓN}

Al cumplirse cada período de almacenaje en cámara de frío, la fruta fue llevada a laboratorio, en donde se realizaron las siguientes evaluaciones. a) Sólidos solubles. Los sólidos solubles se midieron con un refractómetro $\left(0+32^{\circ}\right.$ Brix $)$. Se utilizó una muestra de pulpa de la fruta por cada repetición. Las evaluaciones de sólidos solubles se efectuaron al momento de la cosecha, y al retirar la fruta del almacenamiento en cámara de frío. b) Pérdida de peso. Se registró el peso inicial al ingresar la fruta a la cámara de frío y al retirar la fruta de ésta. Además se registró el peso de la fruta a los 7 y 14 días de permanencia en laboratorio.

\section{ANÁLISIS ESTADÍSTICO}

Los datos obtenidos fueron sometidos a un análisis de contrastes ortogonales, y las medias de los tratamientos y subtratamientos se compararon a través de diferencia de significancia mínima (LSD) $(\mathrm{p} \leq 0,05)$.

\section{RESULTADOS Y DISCUSIÓN}

La deshidratación de la fruta durante la poscosecha se indica en el cuadro 1 . En este sentido el cultivar Berkeley presentó la mayor pérdida de peso al cosechar la fruta por la tarde y con 4 horas de permanencia en huerto, en tanto que los cultivares Brigitta y Elliott no respondieron a los tratamientos ni subtratamientos considerados para la investigación. Con 14 días de almacenaje refrigerado sólo el cultivar Berkeley presenta valores de pérdida de peso que superan al 5,0\%. Según Fundación Chile (1986) y Pino (1992) basta un $5,0 \%$ de deshidratación en la fruta para que ésta logre poca apariencia atractiva.

La mayor pérdida de peso en todos los cultivares se registró en la fruta colectada con 4 horas de permanencia en huerto, esto coincide con lo observado por Belmar (1993) y se explica porque al prolongar el período de permanencia en huerto la fruta se expone al efecto de la alta temperatura y menor humedad relativa, lo que según Claypool (1975) provoca un gradiente hídrico que provoca el incremento de la transpiración en la fruta, lo que causa su posterior deshidratación.

En general, la fruta se mantiene en buenas condiciones entre 7 y 14 días de permanencia en cámara de frío. Este hecho coincide con lo enunciado por Yommi y Godoy (2002), que proponen un almacenaje refrigerado de alrededor de 14 días para mantener la fruta en buenas condiciones.

El contenido de sólidos solubles ( ${ }^{\circ}$ Brix) de la fruta de los cultivares Berkeley, Brigitta y Elliott se indica en el cuadro 2. En la localidad de Freire al momento de la cosecha fue cercano al 13\%. En este sentido, Kushman y Ballinger (1968) proponen niveles superiores a $10^{\circ}$ Brix como requerimiento. Asimismo, Wills et al. (1985), señalan que los arándanos deben cosecharse con niveles de sólidos solubles de 12 a $14^{\circ}$ Brix, para obtener fruta con las características organolépticas deseadas; sin embargo, en la zona Sur de nuestro país los frutos de arándano aparentemente logran el contenido de sólidos solubles antes de adquirir la coloración completa (Moggia, 1991). 


\section{Cuadro 1}

Pérdida de peso promedio (\%) de arándanos cultivar Berkeley provenientes de la localidad de Freire, Región de La Araucanía; luego de 14 días de almacenaje refrigerado y 7 y 14 días de permanencia en laboratorio

\begin{tabular}{|c|c|c|c|c|c|c|c|}
\hline \multirow{3}{*}{$\begin{array}{c}\text { Momento } \\
\text { Cosecha }\end{array}$} & \multirow{3}{*}{$\begin{array}{c}\text { Permanencia } \\
\text { en huerto (horas) }\end{array}$} & \multicolumn{6}{|c|}{$\begin{array}{c}\text { Pérdida de peso promedio (\%) según días en cámara } \\
\text { de frío y ambiente de laboratorio }\end{array}$} \\
\hline & & \multirow{2}{*}{\multicolumn{2}{|c|}{ Frío }} & \multicolumn{4}{|c|}{ Laboratorio } \\
\hline & & & & \multicolumn{2}{|c|}{7} & \multicolumn{2}{|c|}{14} \\
\hline \multirow[t]{2}{*}{ Mañana } & 2 & 5 & a & 23,2 & b B & 34 & b B \\
\hline & 4 & 5,6 & a NS & 13,4 & a A & 23 & a A \\
\hline \multirow[t]{2}{*}{ Tarde } & 2 & 6,4 & $a b$ & 12 & a & 21 & a \\
\hline & 4 & 7,4 & b NS & 13 & a NS & 22 & a NS \\
\hline \multicolumn{2}{|c|}{ Coeficiente de Variación (\%) } & \multicolumn{2}{|c|}{$7,45 \%$} & \multicolumn{2}{|c|}{$10,01 \%$} & \multicolumn{2}{|c|}{$6,20 \%$} \\
\hline \multicolumn{8}{|c|}{ Brigitta } \\
\hline \multirow[t]{2}{*}{ Mañana } & 2 & 4,3 & & 15,9 & & 22,4 & \\
\hline & 4 & 4,6 & NS & 15,2 & NS & 21,6 & NS \\
\hline \multirow[t]{2}{*}{ Tarde } & 2 & 4,2 & & 13,7 & & 21,9 & \\
\hline & 4 & 4,3 & ns NS & 14,8 & ns NS & 23,3 & ns NS \\
\hline \multicolumn{2}{|c|}{ Coeficiente de Variación (\%) } & \multicolumn{2}{|c|}{$4,51 \%$} & \multicolumn{2}{|c|}{$10,85 \%$} & \multicolumn{2}{|c|}{$9,98 \%$} \\
\hline \multicolumn{8}{|c|}{ Elliott } \\
\hline \multirow[t]{2}{*}{ Mañana } & 2 & 3,7 & & 16,8 & & 28 & \\
\hline & 4 & 4,3 & NS & 17 & NS & 28 & NS \\
\hline \multirow[t]{2}{*}{ Tarde } & 2 & 3,8 & & 17,1 & & 28 & \\
\hline & 4 & 4,3 & ns NS & 15,4 & ns NS & 26 & ns NS \\
\hline \multicolumn{2}{|c|}{ Coeficiente de Variación (\%) } & \multicolumn{2}{|c|}{$3,34 \%$} & \multicolumn{2}{|c|}{$5,45 \%$} & \multicolumn{2}{|c|}{$5,45 \%$} \\
\hline
\end{tabular}

Cifras con la misma letra minúscula no difieren significativamente entre tratamientos; y cifras con la misma letra mayúscula no difieren significativamente entre subtratamientos según prueba LSD $(\mathrm{p} \leq 0,05)$.

ns: No significativo para tratamientos.

NS: no significativo para subtratamientos.

En general, el contenido de sólidos solubles $\left({ }^{\circ}\right.$ Brix) en la fruta de los cultivares Berkeley, Brigitta y Elliott aparentemente no depende del momento de cosecha de la fruta ni de la permanencia en huerto a la que ésta es sometida, ya que sólo se encontraron diferencias significativas con respecto a la permanencia en huerto en el cultivar Berkeley al momento de la cosecha, y en el cultivar Brigitta, tras 7 días de almacenaje refrigerado. La uniformidad de los sólidos solubles al momento de la cosecha puede verse influenciado por el color de cubrimiento, principal indicador de madurez de la fruta (Berguer, 1991). Los resultados obtenidos en cuanto a sólidos solubles coinciden con lo reportado por Belmar (1993) en relación a lo observado sobre el contenido de sólidos solubles en los cultivares Berkeley y Elliott.

\section{CONCLUSIONES}

1. Al cosechar la fruta por la tarde se registró un aumento en la deshidratación de la fruta durante la poscosecha, mientras que el contenido de sólidos solubles no registró variación con respecto al momento de cosecha. 
Cuadro 2

Sólidos solubles (valores promedio) medidos al momento de la cosecha y luego de 14 días de mantención en cámara de frío en arándanos cultivar Berkeley, Brigitta y Elliott provenientes de la localidad de Freire, Región de La Araucanía

\begin{tabular}{|c|c|c|c|c|c|}
\hline \multirow{3}{*}{$\begin{array}{l}\text { Momento } \\
\text { de cosecha }\end{array}$} & \multirow{3}{*}{$\begin{array}{c}\text { Permanencia } \\
\text { en huerto (horas) }\end{array}$} & \multicolumn{4}{|c|}{ Sólidos solubles ( ${ }^{\circ}$ Brix) } \\
\hline & & \multirow{2}{*}{\multicolumn{2}{|c|}{ Cosecha }} & \multicolumn{2}{|c|}{$\begin{array}{c}\text { Almacenaje } \\
\text { refrigerado (días) }\end{array}$} \\
\hline & & & & \multicolumn{2}{|c|}{14} \\
\hline \multicolumn{6}{|c|}{ Berkeley } \\
\hline \multirow[t]{2}{*}{ Mañana } & 2 & 13,2 & & 13,8 & \\
\hline & 4 & 13,5 & NS & 13,8 & NS \\
\hline \multirow[t]{2}{*}{ Tarde } & 2 & 13,4 & A & 13,9 & \\
\hline & 4 & 13,7 & ns B & 14,1 & ns NS \\
\hline \multicolumn{2}{|c|}{ Coeficiente de Variación (\%) } & \multicolumn{2}{|c|}{$1.76 \%$} & \multicolumn{2}{|c|}{$8,37 \%$} \\
\hline \multicolumn{6}{|c|}{ Brigitta } \\
\hline \multirow[t]{2}{*}{ Mañana } & 2 & 13,2 & & 13,5 & \\
\hline & 4 & 13,4 & NS & 13,6 & NS \\
\hline \multirow[t]{2}{*}{ Tarde } & 2 & 13,3 & & 14,0 & \\
\hline & 4 & 13,5 & ns NS & 13,9 & ns NS \\
\hline \multicolumn{2}{|c|}{ Coeficiente de Variación (\%) } & \multicolumn{2}{|c|}{$1,88 \%$} & \multicolumn{2}{|c|}{$5,15 \%$} \\
\hline \multicolumn{2}{|r|}{ Elliott } & & & & \\
\hline \multirow[t]{2}{*}{ Mañana } & 2 & 12,8 & & 13,3 & \\
\hline & 4 & 13,1 & NS & 13,5 & NS \\
\hline \multirow[t]{2}{*}{ Tarde } & 2 & 13,0 & & 13,5 & \\
\hline & 4 & 13,3 & ns NS & 13,5 & ns NS \\
\hline \multicolumn{2}{|c|}{ Coeficiente de Variación (\%) } & \multicolumn{2}{|c|}{$2,04 \%$} & \multicolumn{2}{|c|}{$2,70 \%$} \\
\hline
\end{tabular}

Cifras con la misma letra minúscula no difieren significativamente entre tratamientos; y cifras con la misma letra mayúscula no difieren significativamente entre subtratamientos según prueba LSD $(p \leq 0,05)$.

ns: No significativo para tratamientos.

NS: no significativo para subtratamientos.

2. Al incrementar el período de permanencia en huerto se registró un aumento en la deshidratación de la fruta en poscosecha, no registrándose un efecto significativo sobre la incidencia de hongos ni el contenido de sólidos solubles, no obstante el cultivar Berkeley presentó un incremento de sólidos solubles al prolongar el período de permanencia en huerto.

3. La deshidratación aumentó al prolongar el período de almacenaje refrigerado. Con 14 días de almacenamiento en cámara de frío la deshidratación fue menor a 5\% en los cultivares
Brigitta y Elliott, cumpliendo con los requerimientos de exportación. La mayor pérdida de peso en todos los tratamientos se presentó en el cultivar Berkeley $(6,1 \%$, rango 5,0 - 7,4\%), seguido por Brigitta (4,3\%, rango 4,2 - 4,6\%) y Elliott (4,0\%, rango 3,7 - 4,3\%).

4. La permanencia en huerto es un factor determinante en la calidad del arándano en poscosecha, cuya magnitud es dependiente del momento de cosecha; por lo tanto, el control de estos factores disminuye la incidencia de hongos y la deshidratación en poscosecha. 


\section{LITERATURA CITADA}

AUDA, C. 1973. Índices de madurez de frutas. Antecedentes recopilados de investigaciones nacionales y extranjeras. Convenio Corfo-Enafri. Publicación técnica N ${ }^{\mathrm{a}} 5$.

BEATTIE, B.; WADE, N. 1997. Almacenamiento, maduración y manipulación de las frutas. In: Arthey D. y P.R. Ashurst (eds.). Procesado de frutas. Acribia, Zaragoza, España. pp. $43-75$

BELMAR, M. 1993. Incidencia del período entre cosecha y prefrío y momento de cosecha en la calidad e infección por hongos de postcosecha en fruta de arándano alto: (Vaccinium corymbosum L.) cvs. Elliott y Berkeley.

BERGER, H. 1991. Maduración e índices de madurez. En: Primer curso internacional de postcosecha. Departamento de producción agrícola. Centro de estudios de postcosecha. Universidad de Chile. Facultad de Ciencia Agrarias y Forestales. Santiago, Chile. 15-26 pp.

CIREN. 2002. Estudio Agrológico IX Región. Descripción de suelos, materiales y símbolos. Publicación 122.

CLAYPOOL, L.L. 1975. Aspectos físicos del deterioro, Universidad de Chile, Facultad de Agronomía. Santiago, Publicaciones Misceláneas Agrícolas 9: 29-37.

DARNELL, R.L.; STUTTE, G.W.; MARTIN, G.C.; LANG, G.A.; EARLY, J.D. 1992. Developmental physiology of rabbiteye blueberry. Hort. Review 13: 339-405.

FUNDACIÓN CHILE, 1986. Aspectos técnico-económicos en la producción de berries. Departamento Agroindustrial. Publicación técnica $\mathrm{N}^{\circ} 16$.

FUNDACIÓN CHILE, 1989. Mantención de la cadena de frío en productos hortofrutícolas. Informativo agroeconómico. $\mathrm{N}^{\mathrm{o}}$ 1. Febrero 1989. 47-56 pp.

GALLETA, G.; HIMELRICK, D. 1989. Small fruit crop management. Prentice-Hall Press. New Jersey. United States of America. 602 pp.

GUERRERO, J. 1993. Situación fitopatológica de las especies frutícolas cultivadas comercialmente en la IX Región. Frontera Agrícola. 1: 45-50 pp.

KADER, A.A. 1992. Postharvest biology and techonology: an overview. In: Kader, A. (ed.). Postharvest technology of horticultural crops ( $2^{\text {nd }}$ ed.). University of Californiam pub N. 3311. pp. 15-20.
KUSHMAN, L.; BALLINGER, W. 1968. Acid and sugar changes during repening in Wolcott Blueberries. Proceeding of the American Society for Horticultural Science 2: 290-295.

LOBOS, W. 1988. El Arándano en Chile. En: Seminario El cultivo del arándano. INIA Carillanca. Temuco, Chile. 191-202 pp.

MOGGIA, C. 1991. Aspectos de cosecha y postcosecha de arándanos In: Arándano, Seminario internacional Producción comercial y perspectivas económicas. 3-4 de octubre de 1991. Talca, Chile.

OLIVOS, C. 1995. Crecimiento y Maduración del fruto de tres cultivares de arándano ojo de conejo (Vaccinium ashei Reade). Tesis Ingeniero Agrónomo. Universidad de Chile. Facultad de Ciencias Agrarias y Forestales. Santiago. Chile. 62 p.

PELAYO, C.; ELEBER, S.E.; KADERG,A.A. 2001. Postharvest life and flavour quality of three strawberry cultivars kept at $5^{\circ} \mathrm{C}$ in air or air $+201 \mathrm{~Pa}$. Postharvest Biology and Techonology 27 (2): 171-183.

PINO, M. 1992. Comportamiento en almacenaje refrigerado de fruta de once cultivares de arándano alto (Vaccinium corymbosum L.) e incidencia y control de hongos en poscosecha. Tesis Ing. Agr. Universidad de La Frontera. Facultad de Ciencias Agropecuarias y Forestales. Temuco. Chile. 136 p.

SALUNKHE, D.K.; BOLIN, H.R.; REDDY, N.R. 1991. Storage, processing, and nutritional quality of fruit and vegetables. Vol. I: Fresh fruits and vegetables ( $2^{\text {nd }}$ ed.) CRC Press, Florida, USA. 323 p.

SANTIBÁÑEZ, F.; URIBE, J. (1993) Atlas agroclimático de Chile, Regiones VI, VII, VIII y IX. Ediciones de la Universidad de Chile, Santiago, Chile. 99 pp.

SUDZUKI, F. 1983. Cultivo de frutales menores. Editorial Universitaria. Santiago, Chile. $184 \mathrm{p}$

WILLS, R.; LEE, T.; MCGLASSON, W.; HALL, E.; GRAHAN, D. 1985. Fisiología y manipulación de frutas y hortalizas postrecolección. Editorial Acribia. Zaragoza, España. 195 p.

YOMMI, A.; GODOY, C. 2002. INTA Balcarce (Argentina). Arándanos, fisiología y tecnologías de postcosecha. Disponible en http://www.inta.gov.ar/balcarce/info/documentos/agric/posco/fruyhort/arandano.htm: Leído el 31 de marzo de 2007 